\title{
IMPROVEMENT OF VME CONTROL SYSTEM FOR VACUUM DEVICES AND MAGNET POWER SUPPLIES OF THE PLS STORAGE RING*
}

\author{
J. C. Yoon", J. W. Lee, T.-Y. Lee, and S. S. Chang \\ Pohang Accelerator Laboratory, Pohang 790-784,Korea
}

\begin{abstract}
In the Pohang Light Source storage ring, there are 12 VME systems, called VMMIU, in the local control sheds around the storage ring. The VMMIU's control and monitor vacuum devices, gate valves, LCW, Injection system, and magnet power supplies. All vacuum devices and magnet power supplies have local controllers. These controllers are connected to the VME system through RS422/RS232 serial communication. Now we are upgrading software and hardware of these VME systems to enhance device execution speed and communication reliability. In this paper, we describe control structure and scheme of both the current VME system and the upgraded one.
\end{abstract}

\section{INTRODUCTION}

The computer control system of the PLS storage ring has distributed control architecture, with three layers of hierarchy; operator interface computer (OIC) layer, subsystem control computer (SCC) layer, and machine interface unit (MIU) layer. The OIC layer is based on Sun Microsystems' SPARCstation with UNIX (SunOS 4.1.3) and X-terminals. The SCC acts as a data gateway between the OIC layer and the MIU layer. The MIU layer is directly interfaced to individual machine devices for lowlevel data acquisition and control. Both the SCC and MIU layer is based on VMEbus standard with OS-9 real-time operating system. Executable application software modules are downloaded from host computers at the system start-up time. There are 4 SCC's and 24 MIU's. The MIU's and host computers are linked through Ethernet (TCP/IP)[1].

The control system is now very stable and reliable enough to meet our control requirements. However, the control system is continuously being upgraded to accommodate additional control requirements. Recently we had a few additional control requirements. The first is U7 undulator, the first insertion device of PLS, which was installed at the storage ring in July 1997. It required

"Work supported in part by the Korean Ministry of Science and Technology and the POSCO company.

\#Email: jc0927@ postech.ac.kr micron level control of the magnet gap, and also the development of VME application software for IDBPM (Insertion Device Beam Position Monitor) and operator interface software. Next some control requirements of the vacuum gauge controllers were upgraded to increase the vacuum monitoring speed. The third control system change came from the energy ramping of the storage ring. Even though the injection energy from linac is $2 \mathrm{GeV}$, the stored beam energy can be raised up to $2.5 \mathrm{GeV}$ inside the storage ring. The energy ramping procedure needs fast and synchronized control of magnet power supplies. For this purpose, both hardware and software was changed. Finally the timing system was upgraded for the uniform fill pattern of the storage ring, which also needed hardware and software improvement.

\section{SYSTEM CONFIGURATION AND HARDWARE}

There are total 215 magnet power supplies in the storage ring. The magnet power supplies can be divided to unipolar power supplies and bipolar power supplies. Unipolar power supplies are used for bending, quadruple, sextuple, and septum magnets. Bipolar power supplies are used for vertical and horizontal corrector magnets. Each unit of unipolar power supplies has an RS422 serial communication port that is connected to VMMIU. On the other hand, each unit of (bipolar) corrector magnet power supplies has a digital type $\mathrm{I} / \mathrm{O}$ port that is connected to the other machine interface called COMIU.

Vacuum system consists of 124 ion pumps, 36 penning ion gauges, 18 Bayart-Alpert ion gauges, 240 photon-stop temperature sensors, and 15 gate valves. These components have their own local controllers that are interfaced to VMMIU. Each of pumps and gauge controllers has an RS422/RS232 type serial communication port, while each of gate valve controllers has a relay type I/O port. The MIU is directly connected to individual devices and performs the low-level data acquisition. For the real time data acquisition and processes, we chose to use the VME system. Each MIU is a diskless ROM-based system. The ROM contains OS-9 real-time operation system, utility, user-define command, and other necessary application modules.

After the application software upgrade was 
accomplished in a separately prepared machine development system, the execution file is downloaded.

A summary of VME boards used for VMMIU's is shown in Table 1.

Table 1: Summary of VME boards used

\begin{tabular}{|l|c|l|}
\hline \multicolumn{1}{|c|}{ Model } & No. & \multicolumn{1}{c|}{ Description } \\
\hline MVME147S-1 & 12 & SBC,25MHz,4MB DRAM \\
\hline BUS6522II & 12 & MIL-STD-1553B Interface \\
\hline AVME9510-I & 12 & 32 digital input/output, 16ADC \\
\hline ESD-ASIO16 & 24 & 12xRS422, 4xRS232 \\
\hline IP-610 & 2 & 6U IP-Carrier Board \\
\hline IP-Octal & 2 & Industry Pack, 8xRS422 \\
\hline
\end{tabular}

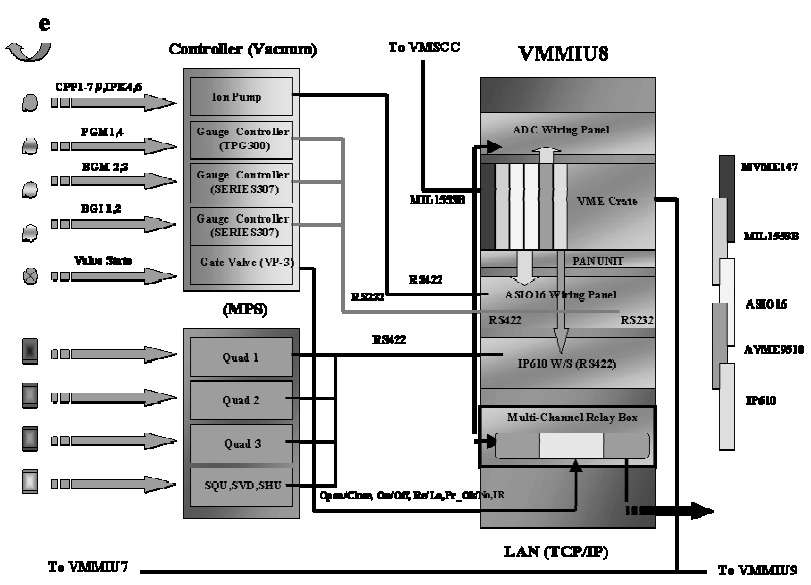

Figure 1: Structure of the VMMIU\#8

\section{SYSTEM UPGRADE}

The computer control system for the remote operation of the U7 undulator is now under development. Development of the VME system for low-level position control and monitoring has been completed. Parker's INDEXER-500 controller was used for the step motor control, and Parker's AR-C absolute encoder was used for the position monitoring. For the motor driving, Parker's PK-130 model was used. A U7 test with local controllers was performed to obtain encoder reference data and to find the locations of the interlock limit switches. The VME control software for position control and monitoring consists of a position control routine and a position monitoring routine. The position control routine directly controls the INDEXER-500 step motor controller. This routine can handle three operator commands: start, normal stop, and emergency stop. The position monitoring routine directly controls the AR-C absolute encoder. This routine can monitor the absolute position of U7. The INDEXER-500 and AR-C are connected to the VME system through an RS232C serial communication. Four limit switches are used for interlocking. If the undulator moves beyond these limit switches, motor driven pulses are immediately stopped. The developed VME control software was installed in the COMIU\#7.

A related work is the development of the VME control system of the IDBPM. For this purpose, one VME ADC I/O board, Acromag's AVME9510-I, was installed in the COMIU\#7. This ADC board receives 15 beam position analog signals (A, B, C, D, AGC, X, Y), two digital output signals (signal level check; TTL level), and four interlock state digital I/O signals, from BPM boards which measure beam positions at the insertion device. The VME control software and the operator interface software were developed, field installed, and the integration test was completed.

For the energy ramping control, two types of VME boards (IP-610, IP-Octal) were installed at the VMMIU\#8 and \#12 for the fast and efficient control of the magnet power supply currents. Also the appropriate VME control software was developed. The structure of the upgraded VMMIU\#8 is shown in Figure 1. Present energy ramping control system has been redesigned and upgraded to reduce ramping time and ensure reliable operation. In a ramping process, the amplitudes of magnetic fields of storage ring magnets should be controlled synchronously for each energy ramping step in order to keep the same optics. This requires the synchronous control of current settings of tens of magnet power supplies distributed in the storage ring. New ramping control system took full advantage of the distributed three-layered PLS storage ring control system to reduce development time and cost. The VME's at the bottom layer, called VMMIU, calculate and control current setting values of magnet power supplies for each ramping step. The VME at an upper layer, called SCC (Subsystem Control Computer), generates synchronization signals for each ramping step. The MIL-1553B field bus, which is already installed for low-level data acquisition control, was used for the synchronization network through which the SCC sends synchronization signals to the lower MIU's. A graphical user interface based on Motif/X-Windows was developed for the operator control of energy ramping

Some control requirements of the vacuum gauge controllers were changed to increase the vacuum monitoring speed. Four Bayard-Alpert ion gauge controllers were installed for insertion device. The 13 cold cathode vacuum gauge controllers have been replaced by Bayart-Alpert ion gauge controllers because of the bad signal caused by either data transmission errors or controller breakdowns. We have improved VME serial boards to resolve the data transmission error problem 
from VME boards to device controllers. The block diagram of the improved serial VME boards is shown in Figure 2. The VMMIU's were upgraded and the VME control software was developed for these changes.

For the uniform fill pattern of the stored beam, the timing control VME system needs to know the stored beam current. One VME ADC I/O board was installed in the timing VME crate. This ADC board receives one analog signal from DCCT that measures the stored beam. The necessary VME software consists of two subroutines; a uniform fill pattern and an arbitrary fill pattern. The present operator interface software was also slightly modified to support the uniform fill pattern operation.

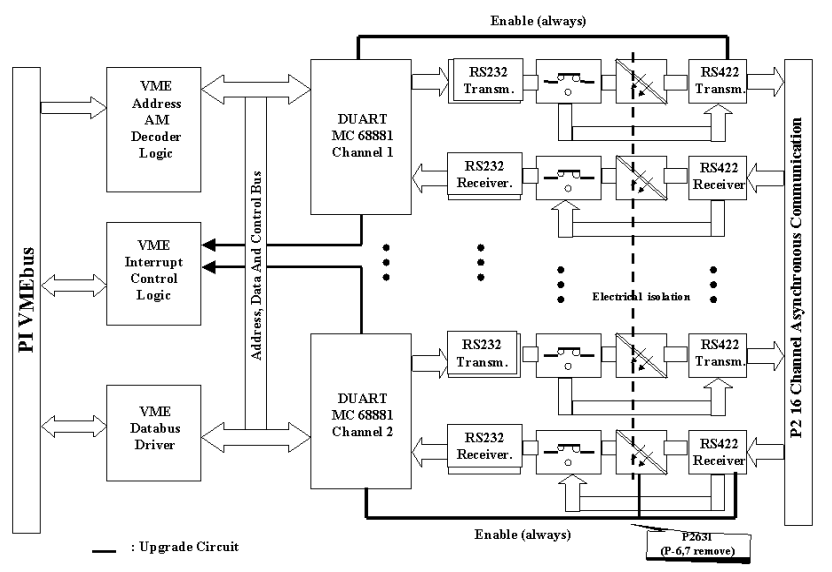

Figure 2: Block Diagram of the improved serial VME board

\section{SOFTWARE UPGRADE}

We chose to use OS-9 $68 \mathrm{~K}$, which is one of the most popular real-time operating systems for VMEbus. In parallel to the hardware structure, the software structure has also the three layer system. There are two important features in the control software; the client/server model to exchange the data between the OIC layer and the SCC layer, and the master/slave model to exchange data between the SCC layer and the MIU layer. The MIU software is basically divided as follows, device access software, application user software, diagnostic monitor software, communication software, and utility software.

Each MIU monitors and controls the software of the corresponding device. Hence, Failure of the application program may lead to the interruption of the related application programs and devices. In this case, the operator should immediately check up the VME computer system and resolve the problem. Moreover, the VME computer often needs to be tested in offline state to upgrade the system or modify the application programs. In order not to stop the running system, we used the dual shared memory module. Shared memory module can provide safe process data, alarm data, and fail device data. The improved control software structure of the VMMIU is shown in Figure 3.

\section{Conclusion}

We are upgrading and modifying the control system to accommodate new control requirements and to apply long-term operational experiences. We are preparing one more duplicated machine development system and operator computer system. This duplicated system will be used for research and development works in the normal operation. In case of main system failure, this system can be used as a backup. Now we have a plan to upgrade the operating system from OS-9 ver. 2.4 to ver. 3.0.3 that contains the Year 2000 Solution. Also, we have a plan to upgrade the control application programs to improve the monitoring refresh time interval of the vacuum pressure.

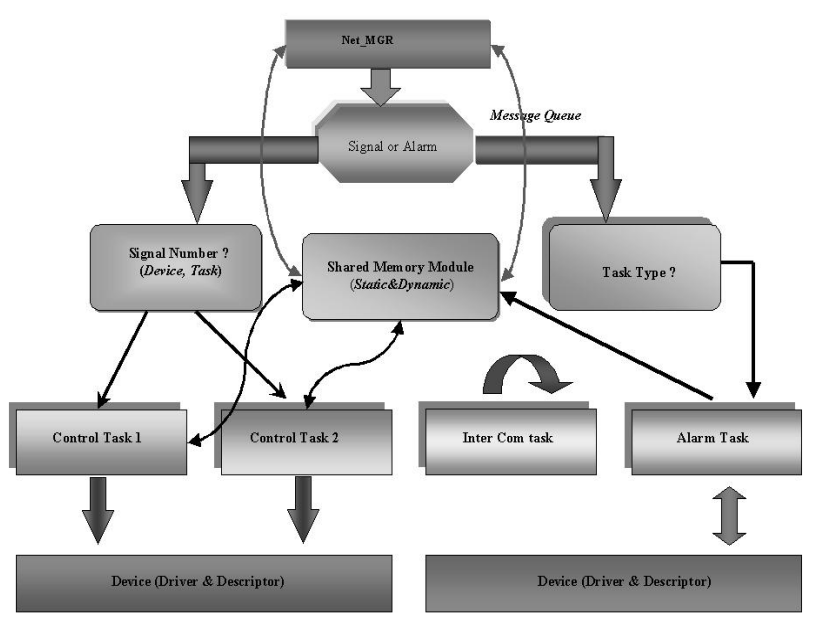

Figure 3: Structure of the upgraded control software

\section{REFERENCES}

[1] I.S.Ko, Jin W.Lee, Jong C. Yun, Eun H. Lee, and Byoung R. Park, "Computer Control System of PLS 2-GeV Storage Ring," IEEE Trans. Nucl. 45 (1998) 2012. 